\title{
Abdominal obesity and circulating metabolites : A twin study approach
}

\section{Bogl, Leonie H.}

2016-03

Bogl , L H , Kaye , S M , Ramo , J T , Kangas , A J , Soininen , P , Hakkarainen , A , Lundbom , J , Lundbom , N, Ortega-Alonso , A , Rissanen, A, Ala-Korpela , M , Kaprio , J \& Pietilainen , K H 2016, ' Abdominal obesity and circulating metabolites : A twin study approach ' , Metabolism, clinical and experimental , vol. 65 , no. 3 , pp. 111-121 . https://doi.org/10.1016/j.metabol.2

http://hdl.handle.net/10138/223885

https://doi.org/10.1016/j.metabol.2015.10.027

publishedVersion

Downloaded from Helda, University of Helsinki institutional repository.

This is an electronic reprint of the original article.

This reprint may differ from the original in pagination and typographic detail.

Please cite the original version. 


\title{
Abdominal obesity and circulating metabolites: A twin study approach
}

\author{
Leonie H. Bogl ${ }^{a, *, 1}$, Sanna M. Kaye $e^{b, 1}$, Joel T. Rämö ${ }^{b, c}$, Antti J. Kangas ${ }^{d, e}$, Pasi Soininen ${ }^{d, e}$, \\ Antti Hakkarainen ${ }^{f}$, Jesper Lundbom ${ }^{f}$, Nina Lundbom ${ }^{f}$, Alfredo Ortega-Alonso ${ }^{c}$, \\ Aila Rissanen $^{b, g}$, Mika Ala-Korpela ${ }^{d, e, h, i}$, Jaakko Kaprio ${ }^{a, c, j, 2}$, Kirsi H. Pietiläinen ${ }^{b, c, k, 2}$ \\ a Department of Public Health, Hjelt Institute, University of Helsinki, Helsinki, Finland \\ ${ }^{\mathrm{b}}$ Obesity Research Unit, Research programs unit, Diabetes and Obesity, University of Helsinki, Helsinki, Finland \\ ${ }^{c}$ Institute for Molecular Medicine FIMM, University of Helsinki, Helsinki, Finland \\ d Computational Medicine, Institute of Health Sciences, University of Oulu, Finland \\ e NMR Metabolomics Laboratory, School of Pharmacy, University of Eastern Finland, Kuopio, Finland \\ ${ }^{\mathrm{f}}$ Helsinki Medical Imaging Center, University of Helsinki, Helsinki, Finland \\ ${ }^{g}$ Department of Psychiatry, Helsinki University Central Hospital, Helsinki, Finland \\ h Oulu University Hospital, Oulu, Finland \\ ${ }^{i}$ Computational Medicine, School of Social and Community Medicine and the Medical Research Council Integrative Epidemiology Unit, \\ University of Bristol, Bristol, UK \\ ${ }^{j}$ Department of Mental Health and Substance Abuse Services, National Institute for Health and Welfare, Helsinki, Finland \\ ${ }^{\mathrm{k}}$ Department of Medicine, Division of Endocrinology, Helsinki University Central Hospital, Helsinki, Finland
}

\section{A R T I C L E I N F O}

Article history:

Received 11 August 2015

Accepted 23 October 2015

Keywords:

Twin study

Bivariate twin model

Genetic correlation

Serum metabolites

Obesity measures

\section{A B S T R A C T}

Objective. To investigate how obesity, insulin resistance and low-grade inflammation link to circulating metabolites, and whether the connections are due to genetic or environmental factors.

Subjects and methods. Circulating serum metabolites were determined by proton NMR spectroscopy. Data from 1368 (531 monozygotic (MZ) and 837 dizygotic (DZ)) twins were used for bivariate twin modeling to derive the genetic $\left(r_{g}\right)$ and environmental $\left(r_{e}\right)$ correlations between waist circumference (WC) and serum metabolites. Detailed examination of the associations between fat distribution (DEXA) and metabolic health (HOMA-IR, CRP) was performed among 286 twins including 33 BMI-discordant MZ pairs (intrapair BMI difference $\geq 3 \mathrm{~kg} / \mathrm{m}^{2}$ ).

Results. Fat, especially in the abdominal area (i.e. WC, android fat \% and android to gynoid fat ratio), together with HOMA-IR and CRP correlated significantly with an atherogenic lipoprotein profile, higher levels of branched-chain (BCAA) and aromatic amino acids, higher levels of glycoprotein, and a more saturated fatty acid profile. In contrast, a higher proportion of gynoid to total fat associated with a favorable metabolite profile. There was a significant genetic overlap between WC and several metabolites, most strongly with phenylalanine $\left(\mathrm{r}_{\mathrm{g}}=\right.$ $0.40)$, glycoprotein $\left(r_{g}=0.37\right)$, serum triglycerides $\left(r_{g}=0.36\right)$, BCAAs $\left(r_{g}=0.30-0.40\right)$, HDL

\footnotetext{
* Corresponding author at: Department of Public Health, PO Box 41, Mannerheimintie 172, University of Helsinki, FI-00014 Helsinki, Finland. Tel.: +358 0504484112.

E-mail address: leonie-helen.bogl@helsinki.fi (L.H. Bogl).

${ }^{1}$ These authors contributed equally to this work.

${ }^{2}$ Shared senior authorship.
} 
particle diameter $\left(r_{g}=-0.33\right)$ and HDL cholesterol $\left(r_{g}=-0.30\right)$. The effect of acquired obesity within the discordant MZ pairs was particularly strong for atherogenic lipoproteins.

Conclusions. A wide range of unfavorable alterations in the serum metabolome was associated with abdominal obesity, insulin resistance and low-grade inflammation. Twin modeling and obesity-discordant twin analysis suggest that these associations are partly explained by shared genes but also reflect mechanisms independent of genetic liability.

(c) 2015 Elsevier Inc. All rights reserved.

\section{Introduction}

Obesity is often accompanied by a cluster of metabolic abnormalities including insulin resistance, atherogenic dyslipidemia and chronic low-grade inflammation. Although body mass index (BMI) predicts the incidence of diabetes and coronary heart disease [1], it cannot by itself identify obese individuals who remain metabolically healthy and normal weight individuals who present disturbed lipid or glucose metabolism and increased cardiovascular risk [2,3]. The assessment of body fat distribution can further improve the evaluation of the subject's metabolic risk. Indeed, observational studies have shown that abdominal obesity, especially excess visceral [4,5], together with liver fat [3] accumulation is the main driver of cardiometabolic risk factors and disease independently of BMI. Traditionally, serum lipids, glucose and insulin were used as markers of cardiovascular risk. More recently, the focus has widened to cover a more global serum metabolomics profile, which has predicted the incidence of cardiovascular events [6], type 2 diabetes [7] and allcause mortality [8] in prospective cohort studies.

Both obesity and serum metabolomics profile are heritable. Genetic factors explain $47 \%-90 \%$ of the interindividual variation in BMI with the remaining variance being attributable to environmental sources and measurement error [9]. Heritability estimates from direct measures of whole-body and regional body fat assessed by dual-energy X-ray absorptiometry (DEXA) are generally similar to the estimates obtained for BMI $[10,11]$. Genetic and environmental influences on serum metabolite levels were recently described in the Finnish twin cohort. Heritability estimates were moderate and ranged between $23 \%$ and 55\% for amino acids and other small-molecule metabolites and were higher for serum lipid (range: 48\%-62\%) and lipoprotein (range: 50\%-76\%) concentrations demonstrating a genetic basis for individual differences in serum metabolite levels [12].

Given these high heritabilities and the significant association between adiposity and metabolic traits [13], a question of interest is whether associations result from potential causal mechanisms or are confounded by shared genes acting pleiotropically on both phenotypes. A recently published Mendelian randomization study suggests causal adverse effects of adiposity with multiple cardiometabolic risk markers in adolescents and young adults from four population-based cohorts in Finland [13]. Obesity discordant twin analyses and bivariate twin modeling are also well suited to explore the extent to which genetic and potential causal environmental factors explain observed associations. Previous twin studies have documented a moderate overlap of both genetic and unique environmental factors that contribute to adiposity and lipid traits $[14,15]$. However, to the best of our knowledge, they have not yet been extended to include a more comprehensive set of circulating metabolites. Thus, in this study of healthy twins, we aimed to 1) estimate the extent of genetic and environmental overlap between waist circumference (WC) and the serum metabolome using bivariate twin modeling techniques; 2) investigate which adiposity and insulin resistance measures are most strongly associated with the serum metabolic profile, including lipids, fatty acids (FAs), and amino acids in twin individuals; 3) examine whether these associations are independent of genetic and familial influences, by conducting within-pair analysis in monozygotic (MZ) twins.

\section{Research Design and Methods}

\subsection{The Twin Cohorts}

The sample was derived from two population-based cohorts, FinnTwin16 (FT16) and FinnTwin12 (FT12) [16]. Both are longitudinal studies of behavioral development and health habits of Finnish twins enrolled during adolescence and repeatedly assessed by self-report questionnaires. The FT12 study includes five consecutive birth cohorts of Finnish twins born in 1983-1987. The questionnaires were sent to twin individuals at age 12 and subsequent follow-up assessments were made when the twins were aged 14, 17 and as young adults (mean age 22 years). The FT16 study includes five consecutive birth cohorts of Finnish twins born in 1975-1979. The questionnaires were sent to the twin individuals at age 16 and subsequent follow-up assessments were made when the twins were aged 17, 18.5, 25 and $~ 34$ years. For both the FT12 and FT16, the baseline and follow-up assessments included surveys of health-related behaviors, anthropometric characteristics, symptom checklists, and social relationships. Zygosity was determined initially by a validated questionnaire method and then confirmed by genetic analysis of polymorphic markers at the Paternity Testing unit, National Institute for Health and Welfare, Helsinki, Finland.

The data presented in this article were derived from a clinical assessment for twins selected from both FT12 and FT16 after the fourth wave questionnaire collection. Pregnant women and subjects on cholesterol-lowering drugs were excluded. Our study population for quantitative genetic analysis included 1368 subjects (FT12: $\mathrm{n}=725$, FT16: $\mathrm{n}=543$ and TwinFat, see below: $n=100 ;$ MZ: 531, DZ: 837; age range: 21.0-31.5; 52.5\% female). A venous blood sample for serum metabolite (NMR) analyses was taken in the morning of the assessment. Height was measured to the nearest millimeter 
with a calibrated stadiometer without shoes and weight to the nearest $100 \mathrm{~g}$ with a calibrated scale wearing light indoor clothing. WC was measured to the nearest millimeter midway between the spina iliaca superior and the lower rib.

\subsection{TwinFat - the Sub-sample With Detailed Adiposity Measures}

The TwinFat sample was enrolled from the FT12 and FT16 cohorts based on the twins' BMI at the fourth wave of the data collection. Twins were selected with the aim of covering the full BMI range of both normal-weight and obese subjects and a full range of within-pair differences in BMI. In addition, 100 twin individuals were chosen at random with respect to BMI (and included in the quantitative genetics analysis sample described above). The Twinfat subsample consisted of 286 subjects (MZ: 136 DZ: 150; age range: 22.8-36.2; 52.9\% female). $33 \mathrm{MZ}$ twin pairs were discordant for BMI (within-pair difference $(\Delta)$ in body mass index $\geq 3 \mathrm{~kg} / \mathrm{m}^{2}$; age range: $22.8-$ $36.1 ; 64 \%$ female). One twin had type 2 diabetes and used metformin and insulin. Another obese co-twin had inactive ulcerative colitis and used mesalazine and azathioprine. All other participants were healthy (based on medical history, clinical examination, and structured psychiatric interview), were normotensive, and did not use any medications except oral contraceptives. Their weights had been stable for at least 3 months prior to the study. Zygosity was confirmed by genotyping of ten informative genetic markers [14].

Body composition was measured by DXA (Lunar Prodigy, Madison, WI, software version 8.8) providing both total body data and regional results (android, gynoid, arms, legs). Fat percentage was calculated as fat mass/(fat mass + lean mass + bone mineral content) for the total body and android and gynoid fat mass and fat percentage were determined from a total body scan as described by Wiklund et al. [17]. Venous blood samples were drawn after a 12-h overnight fast for the measurement of serum metabolites (NMR), glucose (measured using the spectrophotometric hexokinase and glucose-6-phosphate dehydrogenase; Roche Diagnostics, Basel, Switzerland), serum insulin (timeresolved immunofluorometric assay; Perkin Elmer, Waltham, MA, USA) and high sensitivity C-reactive protein (CRP) (Cobas CRP (Latex)HS, Roche Diagnostics). HOMA-IR was calculated as glucose*insulin/22.5 [18].

A subsample of the TwinFat, $84 \mathrm{MZ}$ subjects (TwinFat Intensive, age range: $22.8-36.2,52 \%$ female) provided magnetic resonance imaging (MRI) measurements for subcutaneous (sc) and intra-abdominal (ia) fat volumes and liver fat content by proton magnetic resonance spectroscopy (MRS) as previously described [19].

Data collection and analysis were approved by the ethics committee of the Department of Public Health of the University of Helsinki, the Institutional Review Board (IRB) of Indiana University and the Helsinki University Central Hospital. All subjects provided written informed consent.

\subsection{The NMR Metabolomics Platform}

All serum samples were analyzed using the same highthroughput NMR metabolomics platform. The sample preparation and NMR spectroscopy methods have been described in detail elsewhere $[20,21]$. The NMR metabolomics methodology provides quantitative information on lipoprotein subclass and particle concentrations, serum FAs including, e.g. omega-3 and omega-6 FAs and low-molecular-weight metabolites such as amino acids, 3-hydroxybutyrate, and glycoprotein. The total number of metabolites analyzed in the current study was 56 . These measures describe the main metabolic pathways. A small number of subjects $(<2 \%)$ have missing values for some metabolites due to rejection of these values by automatic sample and measurement quality control.

\subsection{Statistical Analyses}

Descriptive characteristics of the study samples were expressed as mean $\pm \mathrm{SE}$. Anthropometric measures and serum metabolite concentrations were standardized by age, sex and cohort and rank-transformed to normality prior to statistical analysis. At first, we used the entire sample treating twins as individuals while accounting for twin pair clustering by survey methods [22]. Pearson correlations were used to determine correlations between body composition and serum metabolites. In an initial analysis in which variables were only standardized by age and cohort and not by sex, the correlation coefficients were very similar for men and women. The confidence intervals for the phenotypic correlations between WC and serum metabolites in men and women were wide and overlapping for most variables. In addition, the interaction terms between sex and each metabolite in the prediction of WC were tested and not significant for 54 out of the tested 56 metabolites. Based on these results, we decided to present the results for men and women combined in order to increase the statistical power and to simplify the presentation of the results.

Next, we performed within-pair analyses in MZ twins to examine how differences in body composition are related to differences in metabolites between co-twins. Within-pair differences $(\Delta)$ of all measures were calculated by subtracting the leaner co-twins' BMI measures from the ones of heavier co-twins', irrespective of the magnitude of the intrapair difference in weight. Likewise, Pearson correlation analyses were performed to examine how $\Delta$ body fat distribution is related to $\Delta$ in serum metabolites. The non-parametric Wilcoxon signed-rank test for matched samples was used to compare the untransformed metabolic values between BMIdiscordant co-twins. Because MZ twins raised together share their genetic material (i.e. are identical at the DNA sequence level) and a common rearing environment, any observed associations between obesity measures and the serum metabolic profile are fully controlled for unmeasured confounding by genetic and shared environmental effects.

In individual-level analysis, 16 principal components, and in within-pair analysis 15 principal components explained more than $95 \%$ of the variance in serum metabolite concentrations. This number was used to correct for multiple testing using the Bonferroni method. The statistical analyses were performed using the Stata statistical software (release 11.0; Stata, College Station, TX) and the rank-transformation to normality was conducted in the statistical software $\mathrm{R}$ version 2.15.0 (package GenABEL). Quantitative genetic modeling to estimate these genetic and environmental variance and 
covariance components is standard in twin studies [23] and described in more detail in the electronic Supplementary material (ESM) Methods and ESM Figure.

\section{Results}

Descriptive characteristics of our study cohorts are shown in Table 1.

\section{Abdominal Obesity and Serum Metabolites: Bivariate Models}

The sex-, age- and cohort-adjusted phenotypic correlations between WC and circulating serum metabolites in individual twins are presented in Fig. 1. The actual Pearson correlation coefficients are shown in ESM Table 1.

\subsection{Phenotypic Correlations}

WC was significantly correlated with 50 out of the 56 investigated serum metabolites towards an unfavorable metabolic profile. Specifically, abdominal obesity was positively correlated with concentrations of triglycerides and very low-density lipoprotein (VLDL) particles, low-density lipoprotein (LDL) particles, and concentrations of small high-density lipoprotein (HDL) particles, as well as total cholesterol, LDL-C, intermediate density lipoprotein cholesterol (IDL-C), Apolipoprotein (Apo) B and the ApoB to ApoA1 ratio. Concentrations of large HDL particles were negatively correlated with WC. Serum FAs as a proportion of total FAs were inversely, albeit weakly correlated with WC, except for the positive correlation with monounsaturated fatty acids (MUFAs). Branched chain amino acids (BCAA; valine, leucine, isoleucine), two aromatic amino acids (phenylalanine and tyrosine), as well as alanine

Table 1 - Characteristics of the study cohorts.

FinnTwin Twinfat Twinfat MZ

\begin{tabular}{llll} 
& $\mathrm{n}=1368$ & $\mathrm{n}=286$ & $\mathrm{n}=136$ \\
Age (yrs) & $24.3 \pm 0.1$ & $28.7 \pm 0.2$ & $29.4 \pm 0.5$ \\
Monozygotic & 531 & 136 & 136 \\
Dizygotic, same sex & 837 & 150 & $\mathrm{NA}$ \\
BMI (kg/m ${ }^{2}$ ) & $23.6 \pm 0.1$ & $25.4 \pm 0.3$ & $26.5 \pm 0.6$ \\
Waist (cm) & $80.9 \pm 0.4$ & $87.3 \pm 0.9$ & $88.3 \pm 1.4$ \\
Total fat (\%) & NA & $29.0 \pm 0.8$ & $31.1 \pm 1.3$ \\
Android fat (\%) & NA & $35.0 \pm 0.9$ & $37.2 \pm 1.3$ \\
Gynoid fat (\%) & NA & $29.9 \pm 0.8$ & $38.5 \pm 1.3$ \\
Android/gynoid fat ratio & NA & $1.0 \pm 0.0$ & $1.0 \pm 0.0$ \\
Android fat/Total fat\% & NA & $1.2 \pm 0.0$ & $1.2 \pm 0.0$ \\
Gynoid fat/Total fat\% & NA & $1.3 \pm 0.0$ & $1.3 \pm 0.0$ \\
Subcutaneous fat (cm $\left.{ }^{3}\right)^{a}$ & NA & NA & $4411.2 \pm 271.9$ \\
Intra-abdominal fat $\left(\mathrm{cm}^{3}\right)^{2}$ & NA & NA & $1166.5 \pm 110.2$ \\
Liver fat (\%) $^{a}$ & NA & NA & $2.9 \pm 0.6$ \\
Glucose (mg/L) & NA & $5.1 \pm 0.0$ & $5.2 \pm 0.1$ \\
Insulin (mU/L) & NA & $6.2 \pm 0.3$ & $6.0 \pm 0.4$ \\
Homa IR & NA & $1.4 \pm 0.1$ & $1.4 \pm 0.1$ \\
hsCRP (mg/L) & NA & $1.7 \pm 0.2$ & $2.0 \pm 0.3$ \\
& & & \\
\hline
\end{tabular}

Values are mean \pm SE. MZ, monozygotic; NA, not assessed.

a $\mathrm{n}=84$. were positively and glycine negatively correlated with WC. Among the measures of glycolysis, WC was positively correlated with pyruvate and negatively correlated with citrate. WC also correlated positively with glycerol, which serves as a marker of lipolysis of the triglycerides. The ketone bodies acetate and 3-hydroxybutyrate were weakly and inversely correlated with WC. WC was not significantly correlated with the following metabolites: histidine, glycine, lactate, acetate, creatinine and urea. The largest positive correlation was seen with the ApoB to ApoA1 ratio $(r=0.35)$ followed by glycoprotein $(r=0.33)$ and the largest negative correlation was observed with HDL particle diameter $(r=-0.33)$ followed by the concentration of large HDL particles and the HDL-C to LDL-C ratio ( $r=-0.31$ for both).

\subsection{Genetic and Environmental Correlations}

To further investigate the relationship between abdominal obesity and the circulating serum metabolites, we decomposed these phenotypic correlations into their genetic and environmental components (Fig. 1, ESM Table 1). The significant genetic and environmental correlations which were observed between WC and many metabolites indicate that the genetic and environmental factors that influence WC overlap partly with those that influence the serum metabolites. However, the strength of the correlations was weak to modest suggesting that only a fraction of the genetic and environmental variation is common to abdominal obesity and the serum metabolites. Overall, we found that genetic and unique environmental correlations were of about the same magnitude as phenotypic correlations for most serum metabolites. Exceptions were the metabolites ApoA1, BCAA and phenylalanine, for which genetic correlations were stronger than unique environmental correlations. In particular, for ApoA1, valine and leucine, genetic correlations were significant $\left(r_{g}=-0.24,0.32\right.$ and 0.36 respectively) but the unique environmental correlations were smaller and did not reach statistical significance. This indicates that shared genetic factors largely explain the significant associations between abdominal obesity and these serum metabolites.

\section{Obesity-related Measures and Serum Metabolites: Detailed Phenotyping in the TwinFat Sub-sample}

Sex- and age-adjusted phenotypic correlations between detailed obesity-related measures and serum metabolites in the TwinFat subsample are presented as a heatmap in Fig. 2. Overall (BMI, \% body fat) and abdominal obesity (WC), android fat\% and android to gynoid ratio correlated positively with an unfavorable lipoprotein profile (i.e. increased VLDL, IDL, LDL and small HDL particle concentrations, IDL- and LDL-C, triglycerides, $A$ poB and $A p o B$ to $A p o A 1$ ratio and reduced large HDL particle concentration, HDL-C and diameter). The strongest correlations were observed between measures of abdominal obesity and small VLDL particle concentration, HDL diameter and triglycerides $(r \geq 0.4)$. Measures of obesity were not significantly correlated with saturated fatty acids (SFAs) but positively with MUFAs $\left(r_{\max }=0.45\right)$ and negatively 


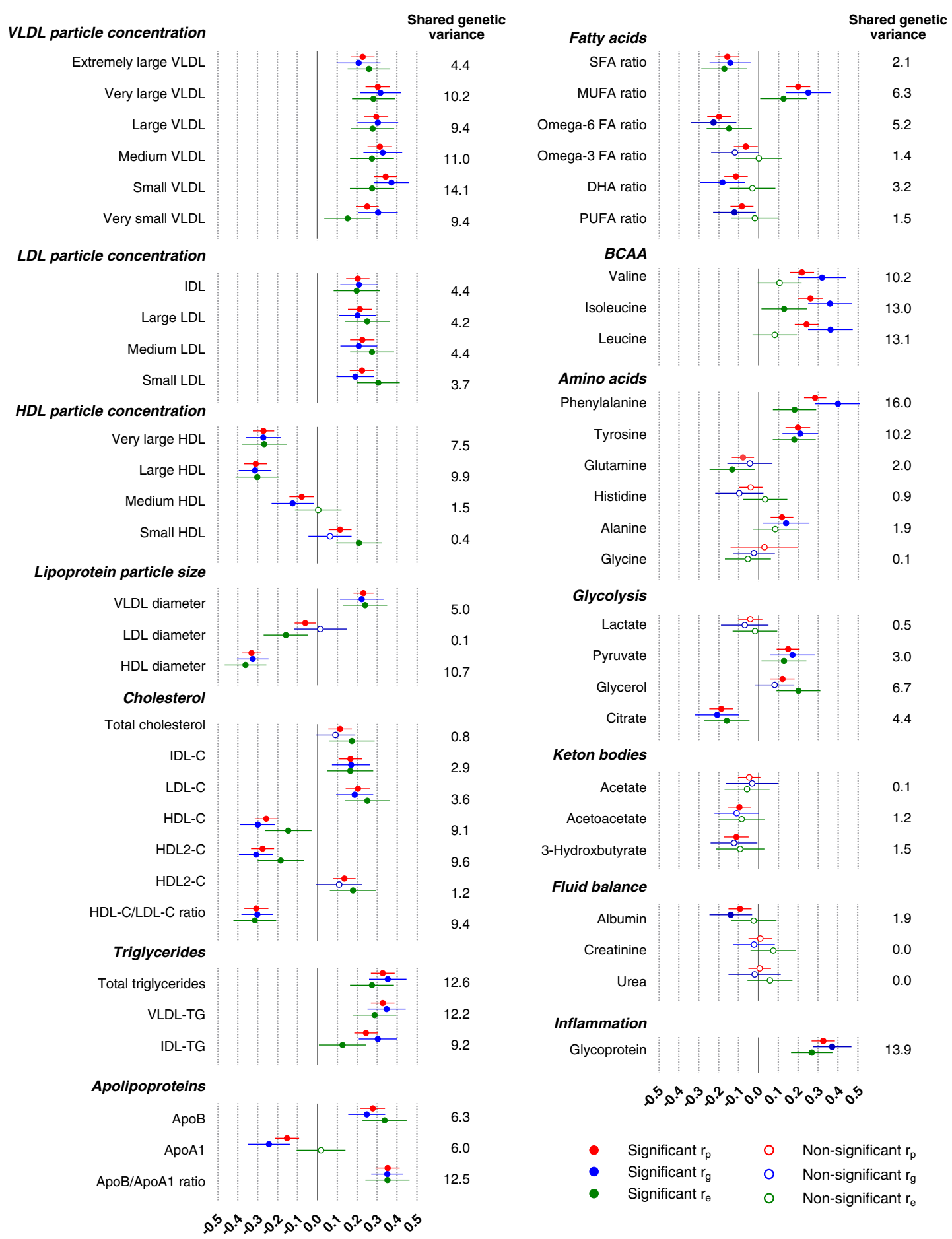

Fig. 1 - Correlations between waist circumference and serum metabolites in 1368 twin individuals. Phenotypic correlations ( $\left.r_{p}\right)$ are shown in red; genetic correlations $\left(r_{\mathrm{g}}\right.$ in blue and unique environmental correlations $\left(\mathrm{r}_{\mathrm{e}}\right)$ in green. The points indicate the correlation coefficient and the lines show the $95 \%$ confidence intervals. All correlations are adjusted for sex, age and cohort. The shared genetic variance was calculated as the $r_{\mathrm{g}}^{2}$. Abbreviations: VLDL, very low-density lipoprotein; LDL, low-density lipoprotein; IDL, intermediate-density-lipoprotein; HDL, high-density-lipoprotein; C, cholesterol; ApoB, Apolipoprotein B; ApoA1, Apolipoprotein A1; SFA, saturated fatty acids; MUFA, monounsaturated fatty acids; FA, fatty acids; DHA, docosahexaenoic acid; PUFA, polyunsaturated fatty acids. The fatty acid ratios indicate the ratio of different classes of fatty acids to total fatty acids.

with polyunsaturated fatty acids (PUFAs) $\left(r_{\max }=-0.56\right)$. Obesity was positively correlated with BCAA and phenylalanine $\left(r_{\max }=0.44, p<0.001\right)$, glycoprotein $\left(r_{\max }=0.47\right)$ and to $a$ weaker extent with tyrosine $\left(r_{\max }=0.27\right)$. BMI and measures of abdominal obesity correlated inversely with glutamine $\left(r_{\max }=-0.26\right)$. Measures of insulin resistance (i.e. fasting 


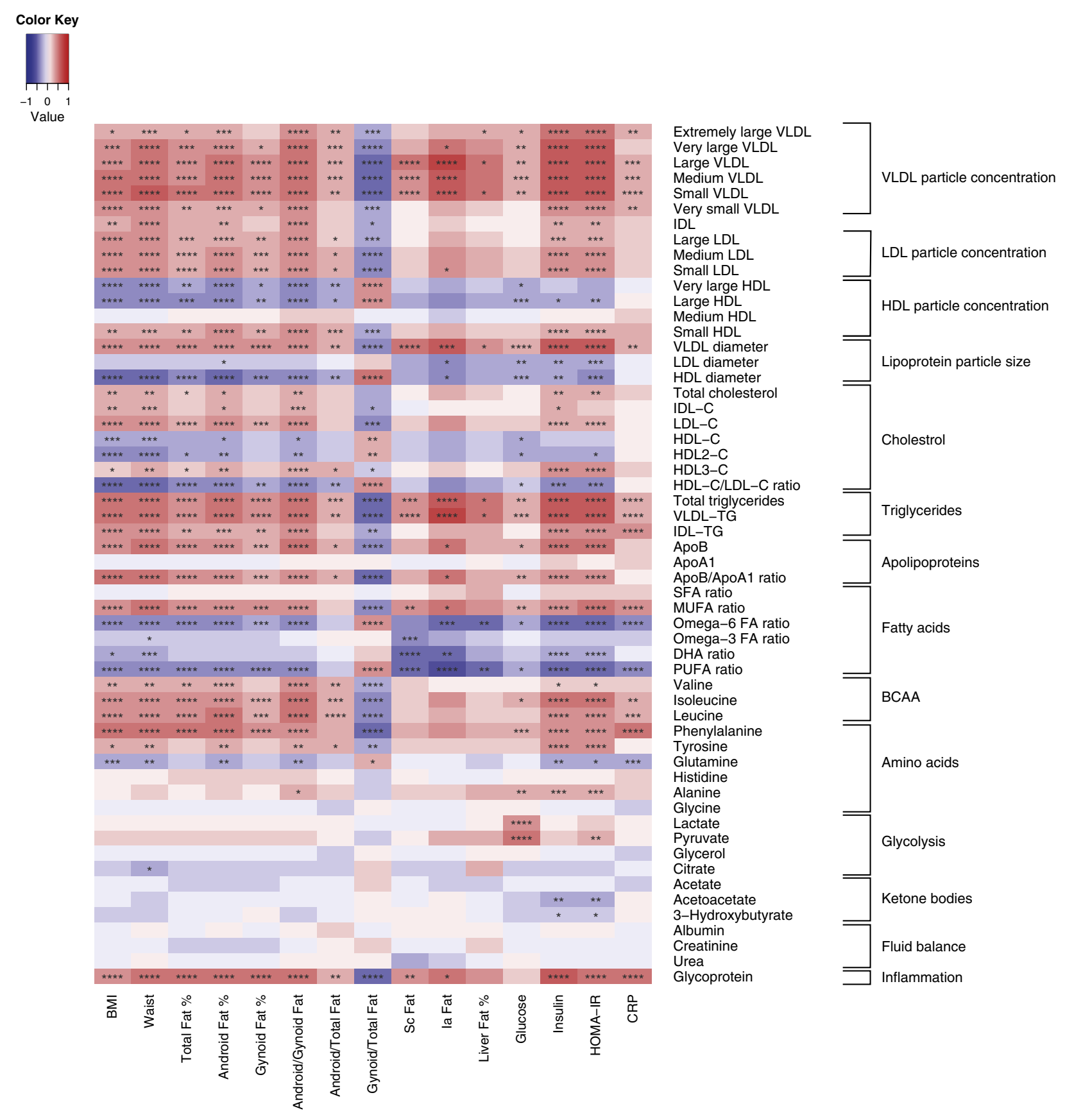

Fig. 2 - Pearson correlations between obesity-related measures and serum metabolites in 286 twin individuals. The sample size for subcutaneous (sc), intra-abdominal (ia) and liver fat was 84. Abbreviations: BMI, body mass index, Waist, waist circumference; HOMA-IR, Homeostatic Model Assessment of Insulin Resistance; CRP, high-sensitive C-reactive protein; VLDL, very low-density lipoprotein; LDL, low-density lipoprotein; IDL, intermediate-density-lipoprotein; HDL, high-densitylipoprotein; C, cholesterol; ApoB, Apolipoprotein B; ApoA1, Apolipoprotein A1; SFA, saturated fatty acids; MUFA, monounsaturated fatty acids; FA, fatty acids; DHA, docosahexaenoic acid; PUFA, polyunsaturated fatty acids; BCAA, branchedchain amino acids. The fatty acid ratios indicate the ratio of different classes of fatty acids to total fatty acids. Android fat and gynoid fat indicate the ratio of fat to total tissue in the android/gynoid areas. The color key denotes the magnitude of the correlation coefficients. All metabolites were rank-transformed and adjusted for sex and age. The P-values denote the statistical significance after correcting for multiple testing: P-values ${ }^{* * * *} \mathrm{p}<0.00000625 ;{ }^{* * *} \mathrm{p}<0.0000625 ;{ }^{* *} \mathrm{p}<0.000625$, ${ }^{*} \mathrm{p}<0.0031$.

insulin and HOMA-IR) showed similar associations to the metabolites as overall and abdominal obesity although many correlations were stronger (e.g. medium VLDL: $r=0.58$, triglycerides: $r=0.56$ and glycoprotein: $r=0.48-0.50$ ).
Additionally, significant inverse correlations were seen between measures of insulin resistance and LDL diameter, acetoacetate and 3-hydroxybutyrate. CRP correlated with an unfavorable metabolic profile and correlations were generally 
weaker than with measures of obesity and insulin resistance, except for the considerable correlation between CRP and glycoprotein $(r=0.48)$. The associations between the ratio of gynoid to total fat mass and the metabolic profile were opposite than with other obesity measures. Correlations were strongest with HDL diameter $(r=0.41)$ and the following metabolites: medium and small VLDL particles, triglycerides, phenylalanine and glycoprotein $(r=-0.43$ to -0.48$)$.

The same analysis that was performed in individual twins was repeated using within-pair differences $(\Delta)$ in MZ twin pairs, thus being able to control for familial and genetic confounding. Compared with analyses performed in twin individuals, there was a notable drop in the strength of most correlation coefficients (Fig. 3). This indicates possible genetic control over the association between obesity measures, insulin resistance, inflammation and the serum metabolic profile. However, exceptions were the following: $\Delta$ android fat $\%$ correlated with lipoprotein profile including $\triangle \mathrm{LDL}-\mathrm{C} \quad(\mathrm{r}=0.46), \quad \Delta \mathrm{LDL}$ particle concentrations $\left(r_{\max }=0.49\right), \Delta L D L$ diameter $(r=-0.41)$ and $\triangle$ ApoB $(r=0.47)$. Similarly, $\Delta$ insulin resistance associated with an unfavorable metabolic profile including $\triangle$ VLDL particle concentration $\left(r_{\max }=0.56\right), \Delta$ triglycerides $(r=0.53), \Delta$ PUFAs $(r=-0.41)$, sisoleucine $(r=0.46)$ and $\Delta$ glutamine $(r=-0.43)$. The correlations between $\Delta \mathrm{ia}$ fat and $\Delta$ liver fat and $\triangle \mathrm{LDL}$ particle concentrations, $\triangle \mathrm{LDL}-\mathrm{C}, \triangle \mathrm{ApoB}, \triangle \mathrm{ApoB}$ to ApoA1 ratio, $\Delta \mathrm{HDL}$ diameter, $\Delta$ acetoacetate, and $\Delta 3$-hydroxybutyrate were stronger than in individual-level analysis. The highest correlation was seen for correlations between $\Delta$ ia fat and $\Delta$ liver fat and the $\Delta A p o B$ to ApoA1 ratio ( $r=0.61$ and 0.58 , respectively). This highlights that acquired accumulation of fat in the abdominal area, and excess ia and liver fat in particular, are important modifiable environmental factors that modify the levels of atherogenic lipoproteins and some other metabolites.

\subsection{BMI-discordant Monozygotic Co-twins}

We further studied the role of environmental factors by comparing the serum metabolites between 33 BMI-discordant MZ twin pairs. The obese co-twins were on average $18 \mathrm{~kg}$ heavier and had $29 \%$ more total fat, $36 \%$ more fat in the android and $17 \%$ more fat in the gynoid area and $280 \%$ more liver fat. In obese co-twins we indeed found significantly increased levels of those metabolites that correlated strongest with obesity measures above (ESM Table 2). Obese co-twins had higher concentrations of VLDL and LDL particles, cholesterol, triglycerides, ApoB, BCAA, lactate, glycerol, urea and glycoprotein than their leaner counterparts. Obese co-twins had lower concentrations of HDL particles, HDL-C and a smaller HDL diameter. The ratios HDL to LDL, ApoB to ApoA1 and PUFA to total FAs were lower in the obese than lean cotwins. The particle concentration of most lipoproteins, HDL particle size and ratios of HDL to LDL and ApoB to ApoA1 remained significantly different between co-twins even after correction for multiple comparison (adjusted $\mathrm{p}<0.0033$ ).

\section{Discussion}

In this study of healthy young adults, abdominal obesity, insulin resistance and low-grade inflammation were associated with higher concentrations of atherogenic lipoproteins, aromatic and BCAA, glycoprotein and lower concentrations of PUFAs and glutamine. The relative contribution of genetic and modifiable environmental factors in explaining these associations was dependent on the metabolite under investigation. Acquired obesity, independent of genetic factors within the MZ BMIdiscordant twin pairs, was most clearly associated with an atherogenic lipoprotein profile.

Previous twin studies have reported that genetic correlations are stronger between physiologically similar phenotypes, such as triglycerides and HDL-C, and weaker for other pairs of phenotypes, such as obesity and blood pressure $[24,25]$ or obesity and lipid traits $[15,26]$. In the present analysis, we extend these earlier observations by quantifying the genetic and environmental factors that underlie abdominal obesity and a large number of metabolites, which are potential novel biomarkers of metabolic diseases. Genetic correlations between abdominal obesity and metabolites were highest for HDL-C and diameter, serum triglycerides, the ApoB to Apoa1 ratio, BCAA, phenylalanine and glycoprotein. Future bivariate linkage and genome-wide association studies are needed to co-localize these correlated traits to a genetic region. As an evidence of a common genetic basis between obesity and serum lipids, previous bivariate linkage studies have reported a pleiotropic quantitative trait locus (QTL) on chromosome 19 which jointly influences serum triglycerides and adiposity [27] and an LDL-C-BMI locus on chromosome 3 [28]. He and colleagues [29] found an inverse association between a genetic risk score for BMI and HDL-C in type 2 diabetic women, which was independent of BMI, indicating that pleiotropic genes could influence both phenotypes through different pathways. Kilpeläinen et al. [30] reported that a locus near IRS is associated with lower body fat percentage and, opposite to what would be expected, with higher insulin resistance and an adverse lipid profile.

Detailed phenotyping of obesity-related measures in a subsample of the twins allowed the investigation of which obesity-related measures were most closely linked to an adverse metabolic risk profile. As previously reported for standard lipid measures [17,31], abdominal obesity and insulin resistance were the strongest correlates of an atherogenic lipoprotein profile including triglycerides and triglycerides in VLDL and IDL, LDL-C, IDL-C and ApoB, VLDL and LDL particle subclasses of all sizes as well as small HDL particle subclasses. Furthermore, obesity correlated negatively with concentrations of large HDL subclasses, HDL-C and HDL-C to LDL-C ratio. This increase in total VLDL and LDL concentration and shift in the lipoprotein subclass distribution towards larger VLDL and smaller HDL and LDL particles are consistent with increased cardiovascular disease risk [32].

In the present study, correlations within MZ twin pairs were generally weaker than in individual-level analysis. This provides evidence of partial confounding due to genetic factors, which is consistent with the finding of significant genetic correlations for abdominal obesity and most metabolites in the quantitative genetic modeling. It is also in agreement with results from large GWAS studies that identified loci and genes associated with these traits [33]. However, measures of abdominal fat distribution (i.e. android fat $\%$ and ia fat), liver fat and insulin resistance were more 


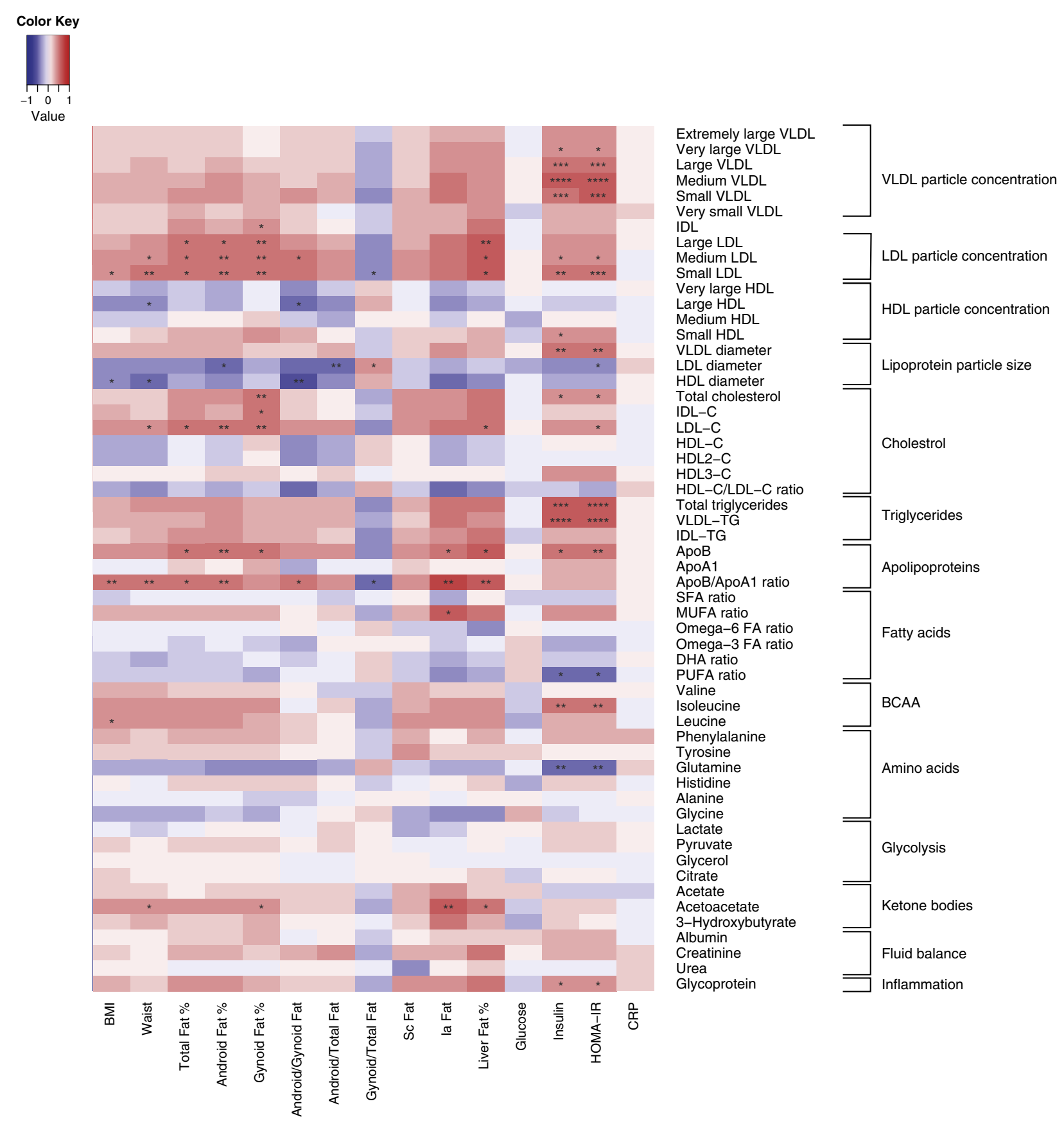

Fig. 3 - Pearson correlations between within-pair differences in obesity-related measures and within-pair differences in serum metabolites in 68 monozygotic twin pairs. The sample size for subcutaneous (sc), intra-abdominal (ia) and liver fat was 42 pairs. Abbreviations: BMI, body mass index, Waist, waist circumference; HOMA-IR, Homeostatic Model Assessment of Insulin Resistance; CRP, high-sensitive C-reactive protein; VLDL, very low-density lipoprotein; LDL, low-density lipoprotein; IDL, intermediate-density-lipoprotein; HDL, high-density-lipoprotein; C, cholesterol; ApoB, Apolipoprotein B; ApoA1, Apolipoprotein A1; SFA, saturated fatty acids; MUFA, monounsaturated fatty acids; FA, fatty acids; DHA, docosahexaenoic acid; PUFA, polyunsaturated fatty acids; BCAA, branched-chain amino acids. The fatty acid ratios indicate the ratio of different classes of fatty acids to total fatty acids. Android fat and gynoid fat indicate the ratio of fat to total tissue in the android/gynoid areas. The color key denotes the magnitude of the correlation coefficients. All metabolites were rank-transformed and adjusted for sex and age. The P-values denote the statistical significance after correcting for multiple testing: $\mathrm{P}$-values ${ }^{* * * *} \mathrm{p}<0.000006667$; ${ }^{* * *} \mathrm{p}<0.00006667 ;{ }^{* *} \mathrm{p}<0.0006667,{ }^{*} \mathrm{p}<0.0033$.

strongly related to VLDL particle concentration, ApoB, LDL-C and LDL diameter in within-pair than in individual-level analysis. Similarly, the heavier co-twins of MZ twin pairs had
$280 \%$ more liver fat and a more atherogenic lipoprotein profile even after correcting for multiple testing. The well-known physiologic role of liver in the lipoprotein metabolism 
suggests that liver fat in our twins is causally linked to the higher VLDL, LDL, and ApoB concentrations. In accordance, lipoprotein kinetic studies in humans have shown that liver fat drives the overproduction of large VLDL particles and ApoB, which in turn initiates a number of lipoprotein changes that increase the risk of atherosclerosis, including hypertriglyceridemia, an increase in small, dense LDL particles and low HDL-C [34].

We found that obesity and insulin resistance were associated with increased concentrations of MUFAs and lower concentrations of PUFAs, in particular omega- 6 FAs and to a lesser extend omega-3 FAs. The serum FA profile is influenced by dietary fat intake over the past few weeks [35], and we have previously reported a lower proportional dietary PUFA intake in the heavier co-twins of the discordant MZ pairs [36]. Rate-limiting enzymes are also important determinants of the serum FA profile; in particular stearoyl-CoA desaturase-1 (SCD-1) synthesizes MUFA from SFA and D6desaturases synthesizes highly unsaturated FAs. Subjects with obesity or metabolic syndrome have increased activity of SCD-1 and D6-desaturase [37], which corresponds well with our finding of more MUFAs and less PUFAs in the serum of obese subjects. In the present study, fasting insulin and HOMA-IR remained significantly correlated with lower concentrations of PUFAs in analysis that controlled for potential confounding by familial factors of genes and shared environment within MZ twins. In two Finnish studies, low concentrations of serum omega-6 PUFAs were prospectively associated with the incidence of impaired fasting glycemia, diabetes mellitus [38] and metabolic syndrome [39].

In our healthy twin sample of young adults, abdominal obesity and insulin resistance were the strongest correlates of BCAA, phenylalanine and tyrosine. It has been known for many years that obesity is associated with an increase in these amino acids $[7,40]$. More recently, it has been shown that obesity blunts amino acid metabolism in adipose tissue and profoundly affects BCAA catabolism. In subcutaneous and visceral adipose tissue obtained during surgery, essential amino acids, leucine, glutamine and serine and 2ketoisocaproic acid differed significantly between obese and lean subjects [41]. In our earlier study of MZ twin pairs discordant for obesity, serum levels of insulin secretionenhancing BCAA were increased in obese male co-twins and adipose tissue transcription profiles exposed significant down-regulation of genes involved with mitochondrial BCAA catabolism $[3,42]$ indicating that the increase in plasma BCAA levels may be due to their decreased oxidation in the peripheral tissues. Our finding of an inverse association between glutamine and insulin resistance is in accordance with previously published data from the Framingham Heart Study and the Malmö Diet and Cancer Study [43]. In the present study we highlight the complexity in the association between obesity and amino acid metabolism, which involves both genetic and environmental factors.

Measures of obesity, insulin resistance and CRP correlated with glycoprotein, an acute-phase protein that is elevated in response to inflammation and infection [44]. Alpha-1 acid glycoprotein has been prospectively associated with both fasting and postchallenge glucose [45] and with the risk of developing diabetes [46]. In a recent study among participants from the Estonian biobank, alpha-1 acid glycoprotein was the strongest circulating biomarker predicting all-cause mortality after adjusting for conventional risk factors [8].

Among all obesity measures studied, measures of abdominal obesity and the ratio of android to gynoid fat were the strongest correlates of an unfavorable serum metabolic profile. In contrast, correlations with the ratio of gynoid to total fat mass were in the opposite direction, supporting the view that having proportionally more fat stored in the gynoid area is metabolically protective. It has been suggested that gluteofemoral fat functions as a 'metabolic sink' to buffer excess energy, thereby preventing ectopic and visceral fat deposition [47].

Combining men and women in the analysis can be seen as a potential limitation. However, we did not observe significant differences by sex in the associations of obesity and metabolites, and therefore combined men and women in the analysis in order to increase statistical power. A previous large study of obesity and circulating metabolites that included 12,664 adolescents and young adults from four population-based cohorts in Finland found significant sex interactions for some metabolites; however the absolute differences were fairly small [13]. The strengths of the present study include its population-based design, the detailed phenotyping of body composition, the inclusion of large sets of serum metabolites and the use of twins to tease out genetic and environmental factors. Potential shortcomings of the study must also be acknowledged. First, the young adult and Caucasian sample utilized in this study limits the generalization to other populations. Second, the cross-sectional study setting does not provide understanding of the temporal relations, even though the use of the MZ discordant pairs permits control of genetic and early family confounders. Finally, multiple comparison correction and the reduced sample size in MZ within-pair and discordant twin analysis increase the likelihood of false negatives (type II error).

In conclusion, abdominal obesity and insulin resistance are associated with changes in the serum metabolome toward reduced cardiometabolic health. Our results suggest that genes that influence variation in abdominal obesity partly overlap with those that influence variation in serum metabolites. However, genetically identical twins that differ in obesity measures also differ in the serum lipoprotein profile, therefore indicating that obesity may be a modifiable environmental factor that leads to lipid disturbances. Thus, our findings give further credence to public health efforts aiming to prevent weight gain and closely linked metabolic abnormalities.

Supplementary data to this article can be found online at http://dx.doi.org/10.1016/j.metabol.2015.10.027.

\section{Author Contributions}

JK, KHP and AR collected the data and recruited study subjects. LHB and SK researched data and wrote the manuscript. JR and AOA assisted in statistical analyses. PS, AJK and MAK performed NMR analyses. AH, JL and NL measured and interpreted the MRI and MRS data. All authors reviewed and edited the manuscript and approved the final version of the manuscript. KHP is the guarantor of this work and, as such, 
had full access to all the data in the study and takes responsibility for the integrity of the data and the accuracy of the data analysis.

\section{Acknowledgments}

Data collection in FinnTwin16 and FinnTwin12 was supported by the National Institute of Alcohol Abuse and Alcoholism (grants AA-12502 and AA-09203 to R. J. Rose). Grant number 137870 to PS; grants 141054, 265240, 263278 and 264146 to JK; grant 266286 to KHP; Centre of Excellence in Research on Mitochondria, Metabolism and Disease (FinMIT) to KHP (grant 272376), Center of Excellence in Complex Disease Genetics (grants 213506 \& 129680 to JK); all from the Academy of Finland. The Finnish Funding Agency for Technology and Innovation (TEKES) and the Strategic Research Funding from the University of Oulu to MAK. Grants from following Foundations: Sigrid Juselius (MAK), Helsinki University Central Hospital (NL, AH, AR, KHP), Jenny and Antti Wihuri (LHB), Novo Nordisk (KHP), the Orion and Farmos Research (SK), Jalmari and Rauha Ahokas (LHB, KHP), Biomedicum Helsinki (LHB), 1.3 milj. klubi-klubben (LHB), Ida Montin (SK), Paavo and Eila Salonen (SK), the Finnish Foundation for Cardiovascular Research (SK, KHP) and a grant from Central Finland Health District Science committee (SK).

\section{Conflict of Interest}

AJK, PS and MAK are shareholders of Brainshake Ltd, a startup company offering NMR-based metabolite profiling.

\section{REFEREN C ES}

[1] Tirosh A, Shai I, Afek A, Dubnov-Raz G, Ayalon N, Gordon B, et al. Adolescent BMI trajectory and risk of diabetes versus coronary disease. N Engl J Med 2011;364:1315-25.

[2] Romero-Corral A, Somers VK, Sierra-Johnson J, Korenfeld Y, Boarin S, Korinek J, et al. Normal weight obesity: a risk factor for cardiometabolic dysregulation and cardiovascular mortality. Eur Heart J 2010;31:737-46.

[3] Naukkarinen J, Heinonen S, Hakkarainen A, Lundbom J, Vuolteenaho K, Saarinen L, et al. Characterising metabolically healthy obesity in weight-discordant monozygotic twins. Diabetologia 2014;57:167-76.

[4] St-Pierre J, Lemieux I, Vohl MC, Perron P, Tremblay G, Despres JP, et al. Contribution of abdominal obesity and hypertriglyceridemia to impaired fasting glucose and coronary artery disease. Am J Cardiol 2002;90:15-8.

[5] Rexrode KM, Carey VJ, Hennekens CH, Walters EE, Colditz GA, Stampfer MJ, et al. Abdominal adiposity and coronary heart disease in women. JAMA 1998;280:1843-8.

[6] Shah SH, Bain JR, Muehlbauer MJ, Stevens RD, Crosslin DR, Haynes C, et al. Association of a peripheral blood metabolic profile with coronary artery disease and risk of subsequent cardiovascular events. Circ Cardiovasc Genet 2010;3:207-14.

[7] Wang TJ, Larson MG, Vasan RS, Cheng S, Rhee EP, McCabe E, et al. Metabolite profiles and the risk of developing diabetes. Nat Med 2011;17:448-53.
[8] Fischer K, Kettunen J, Wurtz P, Haller T, Havulinna AS, Kangas AJ, et al. Biomarker profiling by nuclear magnetic resonance spectroscopy for the prediction of all-cause mortality: an observational study of 17,345 persons. PLoS Med 2014;11:e1001606.

[9] Elks CE, den Hoed M, Zhao JH, Sharp SJ, Wareham NJ, Loos RJ, et al. Variability in the heritability of body mass index: a systematic review and meta-regression. Front Endocrinol (Lausanne) 2012;3:29.

[10] Hsu FC, Lenchik L, Nicklas BJ, Lohman K, Register TC, Mychaleckyj J, et al. Heritability of body composition measured by DXA in the diabetes heart study. Obes Res 2005;13:312-9.

[11] Malis C, Rasmussen EL, Poulsen P, Petersen I, Christensen K, Beck-Nielsen $\mathrm{H}$, et al. Total and regional fat distribution is strongly influenced by genetic factors in young and elderly twins. Obes Res 2005;13:2139-45.

[12] Kettunen J, Tukiainen T, Sarin AP, Ortega-Alonso A, Tikkanen E, Lyytikainen LP, et al. Genome-wide association study identifies multiple loci influencing human serum metabolite levels. Nat Genet 2012;44:269-76.

[13] Wurtz P, Wang Q Kangas AJ, Richmond RC, Skarp J, Tiainen $\mathrm{M}$, et al. Metabolic signatures of adiposity in young adults: Mendelian randomization analysis and effects of weight change. PLoS Med 2014;11:e1001765.

[14] Pietiläinen KH, Rissanen A, Laamanen M, Lindholm AK, Markkula $\mathrm{H}$, Yki-Järvinen $\mathrm{H}$, et al. Growth patterns in young adult monozygotic twin pairs discordant and concordant for obesity. Twin Res 2004;7:421-9.

[15] Pang Z, Zhang D, Li S, Duan H, Hjelmborg J, Kruse TA, et al. Multivariate modelling of endophenotypes associated with the metabolic syndrome in Chinese twins. Diabetologia 2010; 53:2554-61.

[16] Kaprio J. The Finnish twin cohort study: an update. Twin Res Hum Genet 2013;16:157-62.

[17] Wiklund P, Toss F, Weinehall L, Hallmans G, Franks PW, Nordstrom A, et al. Abdominal and gynoid fat mass are associated with cardiovascular risk factors in men and women. J Clin Endocrinol Metab 2008;93:4360-6.

[18] Matthews DR, Hosker JP, Rudenski AS, Naylor BA, Treacher DF, Turner RC. Homeostasis model assessment: insulin resistance and beta-cell function from fasting plasma glucose and insulin concentrations in man. Diabetologia 1985; 28:412-9.

[19] Kaye SM, Maranghi M, Bogl LH, Kaprio J, Hakkarainen A, Lundbom J, et al. Acquired liver fat is a key determinant of serum lipid alterations in healthy monozygotic twins. Obesity (Silver Spring) 2013;21:1815-22.

[20] Soininen P, Kangas AJ, Wurtz P, Tukiainen T, Tynkkynen T, Laatikainen R, et al. High-throughput serum NMR metabonomics for cost-effective holistic studies on systemic metabolism. Analyst 2009;134:1781-5.

[21] Soininen P, Kangas A, Würtz P, Suna T, Ala-Korpela M. Quantitative serum nuclear magnetic resonance metabolomics in cardiovascular epidemiology and genetics circulation. Cardiovascular genetics 2015;131:774-85.

[22] Rao JNK, Scott AJ. On chi-square tests for multiway contingency tables with cell proportions estimated from survey data. Ann Stat 1984;12:46-50.

[23] Plomin R, DeFries JC, McClearn GE, McGuffin P. Behavioral genetics. 5th ed. New York, NY: Worth Publishers; 2008.

[24] Duan H, Pang Z, Zhang D, Li S, Kruse TA, Kyvik KO, et al. Genetic and environmental dissections of sub-phenotypes of metabolic syndrome in the Chinese population: a twin-based heritability study. Obes Facts 2011;4:99-104.

[25] Benyamin B, Sorensen TI, Schousboe K, Fenger M, Visscher PM, Kyvik KO. Are there common genetic and environmental factors behind the endophenotypes associated with the metabolic syndrome? Diabetologia 2007;50:1880-8.

[26] Pietiläinen KH, Söderlund S, Rissanen A, Nakanishi S, Jauhiainen M, Taskinen MR, et al. HDL subspecies in young 
adult twins: heritability and impact of overweight. Obesity (Silver Spring) 2009;17:1208-14.

[27] Feitosa MF, Rice T, North KE, Kraja A, Rankinen T, Leon AS, et al. Pleiotropic QTL on chromosome 19q13 for triglycerides and adiposity: the HERITAGE family study. Atherosclerosis 2006;185:426-32.

[28] Hasstedt SJ, Hanis CL, Elbein SC, American Diabetes Association GENNID Study Group. Univariate and bivariate linkage analysis identifies pleiotropic loci underlying lipid levels and type 2 diabetes risk. Ann Hum Genet 2010;74: 308-15.

[29] He M, Cornelis MC, Franks PW, Zhang C, Hu FB, Qi L. Obesity genotype score and cardiovascular risk in women with type 2 diabetes mellitus. Arterioscler Thromb Vasc Biol 2010;30: 327-32.

[30] Kilpelainen TO, Zillikens MC, Stancakova A, Finucane FM, Ried JS, Langenberg C, et al. Genetic variation near IRS1 associates with reduced adiposity and an impaired metabolic profile. Nat Genet 2011;43:753-60.

[31] Janssen I, Katzmarzyk PT, Ross R. Waist circumference and not body mass index explains obesity-related health risk. Am J Clin Nutr 2004;79:379-84.

[32] Krauss RM. Lipoprotein subfractions and cardiovascular disease risk. Curr Opin Lipidol 2010;21:305-11.

[33] Kraja AT, Vaidya D, Pankow JS, Goodarzi MO, Assimes TL, Kullo IJ, et al. A bivariate genome-wide approach to metabolic syndrome: STAMPEED consortium. Diabetes 2011;60:1329-39.

[34] Adiels M, Taskinen MR, Packard C, Caslake MJ, SoroPaavonen A, Westerbacka J, et al. Overproduction of large VLDL particles is driven by increased liver fat content in man. Diabetologia 2006;49:755-65.

[35] Arab L. Biomarkers of fat and fatty acid intake. J Nutr 2003; 133(Suppl. 3):925S-32S.

[36] Pietilainen KH, Korkeila M, Bogl LH, Westerterp KR, YkiJarvinen $\mathrm{H}$, Kaprio J, et al. Inaccuracies in food and physical activity diaries of obese subjects: complementary evidence from doubly labeled water and co-twin assessments. Int J Obes (Lond) 2010;34:437-45.
[37] Vessby B. Dietary fat, fatty acid composition in plasma and the metabolic syndrome. Curr Opin Lipidol 2003;14:15-9.

[38] Laaksonen DE, Lakka TA, Lakka HM, Nyyssonen K, Rissanen $\mathrm{T}$, Niskanen LK, et al. Serum fatty acid composition predicts development of impaired fasting glycaemia and diabetes in middle-aged men. Diabet Med 2002;19:456-64.

[39] Vanhala M, Saltevo J, Soininen P, Kautiainen H, Kangas AJ, Ala-Korpela M, et al. Serum omega- 6 polyunsaturated fatty acids and the metabolic syndrome: a longitudinal population-based cohort study. Am J Epidemiol 2012;176:253-60.

[40] Felig P, Marliss E, Cahill Jr GF. Plasma amino acid levels and insulin secretion in obesity. N Engl J Med 1969;281:811-6.

[41] Hanzu FA, Vinaixa M, Papageorgiou A, Parrizas M, Correig X, Delgado $\mathrm{S}$, et al. Obesity rather than regional fat depots marks the metabolomic pattern of adipose tissue: an untargeted metabolomic approach. Obesity (Silver Spring) 2014;22:698-704.

[42] Pietiläinen KH, Naukkarinen J, Rissanen A, Saharinen J, Ellonen P, Keranen H, et al. Global transcript profiles of fat in monozygotic twins discordant for BMI: pathways behind acquired obesity. PLoS Med 2008;5:e51.

[43] Cheng S, Rhee EP, Larson MG, Lewis GD, McCabe EL, Shen D, et al. Metabolite profiling identifies pathways associated with metabolic risk in humans. Circulation 2012;125:2222-31.

[44] Fournier T, Medjoubi-N N, Porquet D. Alpha-1-acid glycoprotein. Biochim Biophys Acta 2000;1482:157-71.

[45] Wurtz P, Tiainen M, Makinen VP, Kangas AJ, Soininen P, Saltevo J, et al. Circulating metabolite predictors of glycemia in middleaged men and women. Diabetes Care 2012;35:1749-56.

[46] Duncan BB, Schmidt MI, Pankow JS, Ballantyne CM, Couper D, Vigo A, et al. Atherosclerosis Risk in Communities Study. Low-grade systemic inflammation and the development of type 2 diabetes: the Atherosclerosis Risk in Communities study. Diabetes 2003;52:1799-805.

[47] Despres JP, Lemieux I, Bergeron J, Pibarot P, Mathieu P, Larose E, et al. Abdominal obesity and the metabolic syndrome: contribution to global cardiometabolic risk. Arterioscler Thromb Vasc Biol 2008;28:1039-49. 